\title{
The Structure of NGC 6309: BRET or Bipolar Outflow?
}

\author{
R. Vázquez ${ }^{1}$, L. F. Miranda ${ }^{2}$, L. Olguín ${ }^{3}$, J. M. Torrelles ${ }^{4}$, J. A. López ${ }^{1}$ \\ ${ }^{1}$ Instituto de Astronomía, UNAM, Ensenada, B. C., Mexico \\ ${ }^{2}$ Instituto de Astrofísica de Andalucía, CSIC, Granada, Spain \\ ${ }^{3}$ Instituto de Astronomía, UNAM, México, D. F., Mexico \\ ${ }^{4}$ Institut d'Estudis Espacials de Catalunya (IEEC/CSIC) and Instituto \\ de Ciencias del Espacio, CSIC, Barcelona, Spain
}

\begin{abstract}
Multiwavelength and multitechnique observations towards NGC 6309 are presented. The data consist of $\lambda 3.6-\mathrm{cm}$ continuum, groundand space-based imaging, and high and low-dispersion longslit spectra. The structure of the central region seems to be a distorted, ring of $20^{\prime \prime}$ in diameter. The lack of [ $\mathrm{NII}]$ emission is conspicuous, as revealed by imaging and low dispersion spectra. Notably, the kinematics of the pointsymmetric (PS) structures of NGC 6309 indicates the presence of lobes or cavities as those generally found in bipolar nebulae. The results lead to conclude that the origin of this peculiar morphology is not related to BRETs, but rather to that of a bipolar PN with PS structures in its lobes.
\end{abstract}

\section{Introduction}

NGC 6309 is a bright planetary nebula (PN) with a point-symmetric (PS) morphology. The extended PS arms in this nebula suggest a BRET (Bipolar Rotating Episodic jet) or some related phenomenon for its formation. As a part of a multiwavelength/multitechnique study of PS planetary nebulae (PNe), we have observed this nebula with the following results.

\section{Observations and results}

$C C D$ imaging: Narrow band CCD direct images were obtained with CAFOS in the 2.2-m telescope at Calar Alto observatory in July 8, 1998. Two filters centered in $\mathrm{H} \alpha(\Delta \lambda=15 \AA)$ and $[\mathrm{NII}] 6583 \AA(\Delta \lambda=20 \AA)$ were used, as well as a CCD Loral $2048 \times 2048$. The scale was $0^{\prime \prime} 33$ pixel $^{-1}$ and the seeing $1^{\prime \prime} \cdot 7$ to $2^{\prime \prime} \cdot 0$.

13.5-cm VLA-D: Radio continuum observations towards NGC 6309 were obtained with the VLA-D in August 8, 1996. A $100 \mathrm{Mhz}$ bandwith and two circular polarizations were used. A marginally resolved source was detected. Mean physical conditions were derived $\left(N_{e}=1500 \mathrm{~cm}^{-3}, M(\mathrm{HII})=0.1 \mathrm{M}_{\odot}\right)$.

HST-WFPC2: Images from the HST Archive were used in order to aid in understanding the general view of the internal morphology of NGC 6309 (Proposal ID: 6119; PI: H. E. Bond). The structure resembles to a distorted ring of approximately $20^{\prime \prime}$ in diamter. 

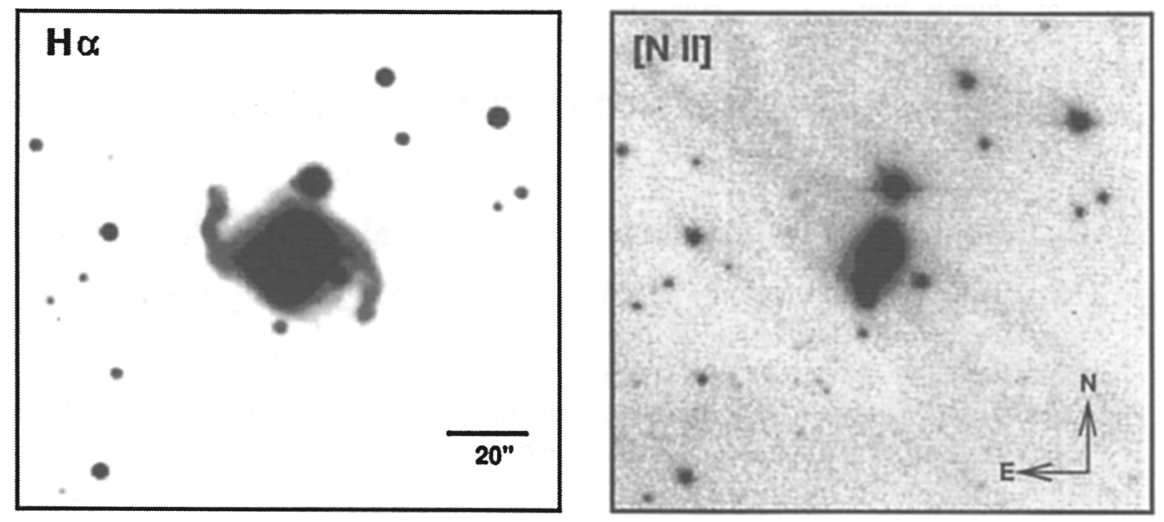

Figure 1. CCD narrow-band images of NGC 6309.

Low-dispersion spectroscopy: Optical spectra were obtained with the B\&Ch spectrometer in the 2.1-m UNAM telescope at the San Pedro Mártir observatory in July 19-20, 1999. A CCD Tek $1024 \times 1024$ was used, as well as a 300 lines/mm grating. Slit width was set to $220-\mu \mathrm{m}\left(1^{\prime \prime} 6\right)$. Spatial and spectral scale is $0^{\prime \prime} 842 \mathrm{pix}^{-1}$ and $4 \AA /$ pix.

High-dispersion spectroscopy: High-dispersion optical spectra were obtained with the Manchester Echelle Spectrometer (MES-SPM) in the 2.1-m telescope at the San Pedro Mártir observatory during May 22-23, 2001. A CCD SITe $1024 \times 1024$ pixels was used as a detector. Slit width was set to $150-\mu \mathrm{m}\left(1^{\prime \prime} 6\right)$. Spatial and spectral scale are $0{ }^{\prime} 6$ pixel $^{-1}$ and $0.1 \AA$ pixel $^{-1}$, respectively.

\section{Concluding remarks}

Imaging shows that the PS features do not emit in [N II], contrary to expectations if they were formed from jet-like ejecta.

Morphology of the central region indicates an inclined and distorted ring whose axis is located at PA $+70^{\circ}$ on the plane of the sky. Assuming that the ring is a circle, the diameter is $\sim 20^{\prime \prime}$ and the inclination angle of the axis with respect to the line of sight is $72^{\circ}$.

The PS features seem to protrude from the extremes of the internal ring instead of the nucleus.

Low-dispersion spectra confirm the high-excitation of the nebula (class 10).

Position-velocity maps from high-dispersion spectra show the presence of cavities or lobes, as seen in bipolar nebulae.

Kinematics seems to be more in agreement with a bipolar outflow than a BRET structure. However, the PS features show apparent knotty structures as those seen in Fleming 1.

Acknowledgments. RV, LO, and JAL were supported by CONACYT (32214-E) and DGAPA-UNAM (IN114199). LFM and JMT were supported by DGESIC (PB98-0670-C02). LO thanks DGEP-UNAM for a graduate scholarship. We acknowledge to the staff of the different facilities used in this research. 